\title{
Development and Evaluation of an In-House ELISA to Detect Anti-FceR1 $\alpha$ IgG Autoantibodies in Chronic Spontaneous Urticaria Patients
}

\author{
Chattip Sripatumtong, ${ }^{1}$ Tunsuda Tansit, ${ }^{1}$ Papapit Tuchinda, ${ }^{2}$ Duangjit Kanistanon, ${ }^{1}$ \\ Kanokvalai Kulthanan $\left(\mathbb{D},{ }^{2}\right.$ and Yuttana Srinoulprasert $\mathbb{C}^{1}$ \\ ${ }^{1}$ Department of Immunology, Faculty of Medicine Siriraj Hospital, Mahidol University, Bangkok, Thailand \\ ${ }^{2}$ Department of Dermatology, Faculty of Medicine Siriraj Hospital, Mahidol University, Bangkok, Thailand \\ Correspondence should be addressed to Yuttana Srinoulprasert; yuttana.sri@mahidol.ac.th
}

Received 25 January 2022; Accepted 12 February 2022; Published 25 February 2022

Academic Editor: Baohui Xu

Copyright (C) 2022 Chattip Sripatumtong et al. This is an open access article distributed under the Creative Commons Attribution License, which permits unrestricted use, distribution, and reproduction in any medium, provided the original work is properly cited.

\begin{abstract}
Background. Association between chronic spontaneous urticaria (CSU) and autoimmunity has been well documented. Autologous serum skin testing could support the autoimmune etiology of CSU, whereas it is difficult to interpret and could not be performed on antihistamine omitted patients. It was found that immunoglobulin $\mathrm{G}$ (IgG) autoantibodies (autoAbs) against high-affinity IgE receptor (FcER1) were suggested as a potential trigger in the pathogenesis of CSU. Although many ELISA protocols have been developed to detect these autoAbs, they lacked validation or a reliable cut-off point. We, therefore, aimed to develop a validated ELISA with a reliable cut-off point to quantitate IgG anti-FceR1 $\alpha$ autoAbs for CSU. Methods. We developed an inhouse ELISA to quantitate IgG anti-FceR1 $\alpha$ autoAbs. Sera from 233 CSU patients and 25 healthy people were used to test with ELISA. The cut-off point was obtained from the results subjected to analyze with receiver operating characteristic (ROC) analysis. ELISA was validated with 116 CSU patients and 150 healthy donors. Results. ELISA revealed that healthy people had a basal level of IgG anti-FceR1 $\alpha$ autoAbs, whereas their levels were significantly lower than autoAbs levels in CSU patients. ROC analysis of ELISA determined the cut-off point at $936.7 \mathrm{ng} / \mathrm{ml}$. Our ELISA was validated and provided excellent sensitivity and specificity at $98.28 \%$ and $92.67 \%$, respectively. Conclusion. Our ELISA could detect significant levels of IgG antiFceR $1 \alpha$ autoAbs in CSU, supporting that these autoAbs were associated with CSU etiology. Our validated ELISA with the reliable cut-off point provided excellent accuracy at $95.11 \%$ (98.28\% sensitivity and $92.67 \%$ specificity). Our ELISA could be an alternative test benefit for the patient who is unable to omit antihistamine treatment.
\end{abstract}

\section{Introduction}

Chronic spontaneous urticaria (CSU) is a dermatological condition characterized by recurring wheal and flares lasting more than six weeks with unrecognized etiology. Several hypotheses of CSU etiology have been proposed, and an autoimmune disorder has been suggested as a possible mechanism [1,2]. Association between CSU and autoreactive antibodies has been revealed and defined as type I and type II hypersensitivity. Type I autoreactive CSU is provoked by immunoglobulin E (IgE) autoantibodies (autoAbs) against self-antigens such as double-strand DNA and thyroid antigens. Type II autoreactive CSU is defined by the effector function of immunoglobulin $\mathrm{G}$ ( $\mathrm{IgG}$ ) against $\mathrm{IgE}$ or the IgE receptor (FceRI) [3, 4]. These autoAbs were discovered in sera of $23 \%-46 \%$ of CSU patients, and they could induce mediator release from normal donor basophils and skin mast cells [4-7]. Interestingly, IgG autoAbs directed against the FceR $1 \alpha$ chain were also detected in $26 \%-77 \%$ of CSU patients from various cohort studies $[6,8-11]$. These 
reports suggested that autoAbs to the alpha-chain of the high-affinity IgE receptor $(\mathrm{FccR} 1 \alpha)$ could be a potential trigger in the pathogenesis of CSU.

The methodology used to detect these autoAbs includes in vivo and in vitro tests. An in vivo test by intradermal injection of a patient's serum into themselves, so-called autologous serum skin testing (ASST), could theoretically support the autoimmune etiology of CSU [7, 12]. ASST was positive in $25 \%-65 \%$ of CSU patients $[6,12,13]$. However, it was difficult to interpret because it provided little or no information about functional components. A significant proportion of patients with allergic or nonallergic rhinitis, multiple drug allergy syndromes, and even healthy control subjects were positive with ASST [14]. Unluckily, ASST could not be done on CSU patients who could not discontinue antihistamine therapy. In vitro techniques such as histamine release (HR) from basophils and western blotting/immunoprecipitation were employed as an alternative method to identify autoAbs $[6,8]$. These laborious procedures are unsuitable for screening purposes and have limited utility for determining autoAbs titers. Many groups of researchers ever developed in-house ELISA, and these results revealed the presence of IgG anti-FceR $1 \alpha$ autoAbs [15-17]. However, how to earn and apply cut-off points was never clearly demonstrated. We, therefore, aimed to develop a validated ELISA with a reliable cutoff point to quantitate IgG anti-FceR $1 \alpha$ autoAbs for CSU serum samples.

\section{Materials and Methods}

2.1. Experimental Design. Sera from 233 CSU patients who attended the Urticaria Clinic (Siriraj Urticaria Center of Reference and Excellence; UCARE), Department of Dermatology, Siriraj Hospital, Bangkok, Thailand, were collected from August 2008 to June 2019. All patients underwent autologous serum skin testing (ASST), and the patients were divided according to the results into the ASST positive and ASST negative groups. Twenty-five healthy individuals without CSU and allergic/autoimmune diseases were also recruited as a control group. These sera from CSU and healthy people were used to find out the cut-off point of the ELISA test. The second cohort of sera was collected from 116 CSU patients who attended the same Urticaria Clinic from August 2018 to June 2021. One hundred and fifty healthy donors were recruited from September 2019 to January 2020. The second cohort group was used to validate the ELISA test. All of the participants in this study provided written informed consent.

2.2. Patient Sample Collection and Preparation for ELISA. Clot blood samples were obtained in sterile conditions from all volunteers. The clot blood samples were subjected to centrifuge at $3000 \mathrm{rpm}$ for 15 minutes at room temperature to obtain sera. The sera were aliquoted and kept at $-20^{\circ} \mathrm{C}$ until use.

2.3. ELISA for Detecting of Human FceR1 $\alpha$ autoAbs. Inhouse enzyme-linked immunosorbent assay (ELISA) was
TABle 1: Demographic data of volunteers.

\begin{tabular}{lccc}
\hline \multirow{2}{*}{ Groups } & \multirow{2}{*}{ Healthy } & ASST + & ASST- \\
\hline Patient no. & 25 & 98 & 135 \\
Age $(\bar{x} \pm S D)$ & $28.4 \pm 7.5$ & $35.7 \pm 12.6$ & $38.3 \pm 12.7$ \\
Gender M/F (\%) & $28.0 / 72.0$ & $18.4 / 81.6$ & $25.9 / 74.1$ \\
\hline
\end{tabular}

developed for detecting anti-FceR $1 \alpha$ auto-antibody. A 96well ELISA plate (Costar, Kennebunk, MC) was coated with $0.00035 \mathrm{mg} / \mathrm{ml}$ of human FceRlalpha protein (Sino Biological, Wayne, PA). After the plate was kept overnight at $4^{\circ} \mathrm{C}$, it was washed five times with PBS-Tween and blocked with 3\% skim milk for 1 hour at $37^{\circ} \mathrm{C}$. After washing again, a $50 \mu \mathrm{l}$ serum sample diluted with $1 \%$ skim milk buffer to final dilution at 1:100 dilution was added into each well; then, the plate was incubated at $37^{\circ} \mathrm{C}$ for one hour. After incubation, the plate was washed; meanwhile, HRP-conjugated rabbit anti-hIgG (Dako, Santa Clara, CA) was freshly prepared final dilution at $1: 1,000$ and added to all sample wells. The plate was incubated at $37^{\circ} \mathrm{C}$ for one hour and washed after all. To measure the amount of anti-FceR $1 \alpha$ auto-antibody, the reaction was developed to detect color using $3,3^{\prime}, 5,5^{\prime}$ -tetramethylbenzidine (Abcam, Cambridge, UK) in substrate solution at room temperature in a dark chamber for $10 \mathrm{~min}$. The reaction was stopped with $1 \mathrm{~N} \mathrm{HCl}$, and optical density (OD) was read at $450 \mathrm{~nm}$ with a microplate spectrophotometer (Biotek, Winooski, VT). To quantify human IgG anti-FceR1 $\alpha$ autoAbs, human immunoglobulin G (hIgG) range from 0.25 to $0.0019 \mu \mathrm{g} / \mathrm{ml}$ was also used to establish a standard curve.

2.4. Statistical Calculations. Both parametric ( $t$-test or paired $t$-test) and nonparametric (sign test or signed-rank test) were used as appropriate. A $p$ value of less than 0.05 was considered to be statistically significant. The amount of hIgG anti-FceR $1 \alpha$ in every sample was calculated from the hIgG standard curve of each corresponding performed experiment. The cut-off value was obtained by applying receiver operating characteristic (ROC) data analysis of hIgG in sera of CSU and healthy donors. Validation of FceR1 $\alpha$ ELISA was performed with 14 CSU-ASST positive, 102 CSUASST negative, and 150 healthy control samples. Values for sensitivity, specificity, positive and negative predictability (PPV and NPV, respectively), and accuracy were calculated.

2.5. Ethical Approvals. This study was approved by the Ethical Committee on Research Involving Human Subjects, Faculty of Medicine, Siriraj Hospital, Mahidol University (COA No. Si 540/2016).

\section{Results}

3.1. Patient Demographics. We recruited two hundred and fifty-eight volunteers, as shown in Table 1. The healthy group contained eighteen females and seven males with a mean age of 39.5 years, the ASST positive group had eighty females and eighteen males with a mean age of 35.7 years, 


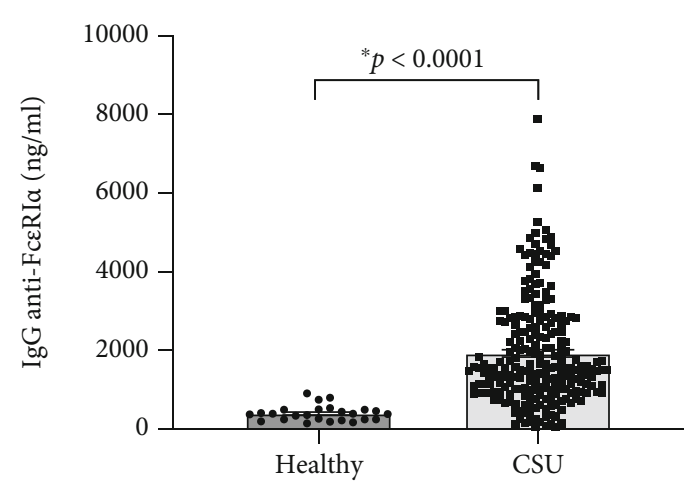

(a)

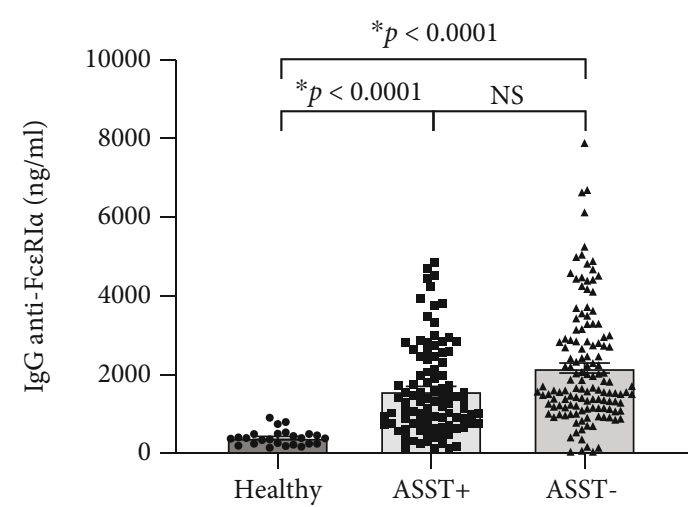

(b)

Figure 1: Overlay of levels of hIgG anti-FceRI $\alpha$ in sera from healthy and CSU donors. (a, b) Bar graphs overlayed with scatter plots represent levels of human IgG against anti-FceR1 $\alpha$ in healthy groups, ASST positive, and ASST negative (mean \pm SEM). *A $p$ value of less than 0.05 was statistically significant.

and the ASST negative group included a hundred females and thirty-five males with a mean age of 38.3 years. The mean age of the three groups was statistically insignificant.


autoAbs. Basal levels of human IgG (hIgG) against the whole molecule of FceR $1 \alpha$ were detected in sera of the healthy group, as shown in Figure 1(a). Sera from CSU contained a higher level of hIgG anti-FceR1 $\alpha$ autoAbs $(1,928.19 \pm 91.48$; mean \pm SEM) as compared with sera from healthy donors $(400.21 \pm 38.90$; mean \pm SEM) (Figure $1(\mathrm{a}))$, as well as the levels of hIgG anti-FceR $1 \alpha$ autoAbs in sera from both ASST positive $(1,589.64 \pm 118.21$; mean \pm SEM $)$ and ASST negative $(2173.95 \pm 128.82$; mean \pm SEM) CSU patients were also significantly higher than that of healthy subjects (Figure 1(b)). However, the levels of hIgG anti-FceR $1 \alpha$ autoAbs among CSU cohorts (ASST positive and ASST negative) were not significantly different even the levels of IgG anti-FceR $1 \alpha$ autoAbs in sera from ASST negative CSU trend to higher than that of ASST positive CSU as shown in Figure 1(b).

3.3. Validation of IgG Anti-FceR1 $\alpha$ ELISA. To obtain cut-off point of hIgG anti-FceR $1 \alpha$ for discrimination between healthy people and CSU patients, levels of hIgG anti-FceR1 $\alpha$ were subjected to analyze by receiver operating characteristic (ROC) analysis. ROC analysis yielded sensitivity and sensitivity of each level of hIgG anti-FceR $1 \alpha$ in two populations for further validation. Youden's index derived from sensitivity and sensitivity of each level of hIgG anti-FceR $1 \alpha$ was demonstrated in Table 2 used to suggest optimal cut-off point. The highest value of Youden's index at 167.30 was chosen to achieve an optimal cut-off value of hIgG anti$\mathrm{FcER} 1 \alpha$ at $936.7 \mathrm{ng} / \mathrm{ml}$.

To validate the cut-off point at $936.7 \mathrm{ng} / \mathrm{ml}$, additional 266 serum samples were from healthy people $(n=150)$; CSU patients (ASST positive, $n=14$, and ASST negative, $n$ $=102$ ) were used to test. Sensitivity, specificity, positive predictive value, and negative predictive value were calculated and revealed at $98.28 \%, 92.67 \%, 91.20 \%$, and $98.58 \%$,
TABLE 2: Youden's index determines cut-off values.

\begin{tabular}{lccc}
\hline Cut off value & Sensitivity & Specificity & Youden's index \\
\hline$>920.0$ & 77.25 & 87.27 & 164.52 \\
$>922.2$ & 77.25 & 89.09 & 166.34 \\
$>927.0$ & 76.82 & 89.09 & 165.91 \\
$>931.2$ & 76.39 & 89.09 & 165.48 \\
$>936.7$ & 76.39 & 90.91 & 167.30 \\
$>942.1$ & 75.97 & 90.91 & 166.88 \\
$>949.7$ & 75.11 & 90.91 & 166.02 \\
$>963.8$ & 74.68 & 90.91 & 165.59 \\
$>978.0$ & 74.25 & 90.91 & 165.16 \\
\hline
\end{tabular}



Figure 2: Levels of hIgG anti-FceRI $\alpha$ in sera from the second cohort of healthy and CSU volunteers. Scatter plot represents levels of human IgG against anti-FceR1 $\alpha$ in healthy $(n=150)$, ASST positive $(n=14)$, and ASST negative $(n=102)\left(\right.$ mean \pm SEM). ${ }^{*} \mathrm{~A} p$ value of less than 0.05 was statistically significant. The cut-off line was set at $936.7 \mathrm{ng} / \mathrm{ml}$. 
TABLE 3: Validation of cut-off value in the second cohort.

\begin{tabular}{lcccr}
\hline & CSU & Healthy & Total & \\
\hline ELISA + & 114 & 11 & 125 & PPV 91.20\% \\
ELISA - & 2 & 139 & 141 & NPV 98.58\% \\
Total & 116 & 150 & 266 & \\
& Sensitivity $98.28 \%$ & Specificity 92.67\% & & \\
\hline
\end{tabular}

${ }^{*}$ Cut-off value was at $936.7 \mathrm{ng} / \mathrm{ml}$.

respectively. The accuracy of the in-house ELISA was at 95.11\%, as shown in Figure 2 and Table 3.

\section{Discussion}

The autoimmune etiology of CSU was corroborated by an in vivo investigation, so-called the autologous serum skin test (ASST), which is an intradermal injection of a patient's serum into their own skin [12]. Wheal and flare reactions indicate a positive result of ASST. Our study found that frequency of ASST positivity in CSU patients was $42 \%$ and $12 \%$ in the first and second cohort studies, respectively. It was consistent with the previous studies that the positive rate of ASST results in CU patients was various (34\%-70\%) [12, 18-22]. Female predominance among CSU cases was also observed in our study and other previous reports [18, 20, 23]. Nevertheless, positive ASST can be observed in clinical remission of CSU patients, allergic and nonallergic patients, autoimmune patients, and even healthy people $[14,24]$. Apart from the difficulty of ASST interpretation, another problematic obstruction to performing ASST is antihistamine omission in some patients. False-positive ASST can occur due to improper preparation of serum samples [24, 25]. Additionally, ASST cannot indicate which factors in serum cause mast cell degranulation. Therefore, identifying factors causing mast cell degranulation in a patient's serum using in vitro test could be an alternative investigation to solve the above issues.

Factors, which circulate in CSU blood and cause mast cell degranulation by induction of wheal and flare reactions in response to ASST, have been demonstrated [26]. These factors can be both IgE dependence and non-IgE dependence. One non-IgE dependent factor was determined as IgG autoAbs against the alpha subunit of the high-affinity FceR1 in sera of CSU patients [6]. It was revealed that human IgG anti-FceR $1 \alpha$ could induce histamine release irrespective of IgE sensitization of the basophils, causing a pathological effect. These autoAbs against $\mathrm{FccR} 1 \alpha$ can provoke chronic stimulation and degranulation of these cells in an IgE-independent fashion [27]. As proof of concept, Hide and colleagues demonstrated that preincubating donor basophils with the soluble fragment of FceR $1 \alpha$ before the addition of purified IgG from sera of patients with CSU was able to neutralize histamine release from basophils. The concept that circulating IgG antibodies against high-affinity IgE receptor FceR $1 \alpha$ likely contribute to the pathogenesis of CSU has become widely accepted [4]. Human IgG autoAbs against FceR $1 \alpha$ can be detected in $24 \%-64 \%$ of sera from CSU patients $[10,15,23,28]$. Interestingly, our study dem- onstrated that the higher prevalence of IgG against FceR $1 \alpha$ was $76.4 \%$ and $98.3 \%$ in the first and second CSU cohorts. Meanwhile, our study also showed $7 \%$ of normal control (NC) sera contained levels of IgG anti-FceR $1 \alpha$ above the cut-off point. The presence of IgG against FceR $1 \alpha$ in NC sera was consistent with previous reports $[23,29]$. A discrepancy of findings could be due to various methods, and the cut-off point in previous studies has never been stated and quantified. Our in-house ELISA was developed and performed with a large cohort to obtain a statistical cut-off point. The newly recruited cohort validated the test with a quantified cut-off point according to the epidemiologic process. Even the presence of FceR $1 \alpha$ autoAbs was noted in the sera of patients with other autoimmune skin conditions and even in healthy subjects, though they did not manifest wheal and flare reactions [15]. However, the levels of FceR $1 \alpha$ autoAbs were below the cut-off in healthy and other autoimmune disorders, as shown in Figures 1 and 2, and Supplement Figure (available here). Our in-house ELISA test could differentiate levels of anti-FceR $1 \alpha$ antibodies in CSU patients from those levels in other autoimmune populations and normal healthy people, which it can be used to screen sera from large patient cohorts. As results from ELISA are informative data, which can be used for further epidemiologic study. The limitation of this research is that ELISA is not a functional test.

Many reports demonstrated an association between the presence of $\operatorname{IgG}$ against $\mathrm{Fc \varepsilon R} 1 \alpha$ and ASST positivity [30-32]. Recently, Ulambayar and colleagues reported that positivity rates for IgG autoAbs to $\mathrm{FccR} 1 \alpha$ were higher in ASST positive CSU patients than in ASST negative CSU patients [23]. Anti-FceR $1 \alpha$ autoAbs were detected in approximately $40 \%$ of CSU sera as well as its higher frequency of positivity in CSU patients was demonstrated in ASST positive sera [23, 33]. As shown in Figure 2 and Table 3, we found that significant levels of IgG against FceR $1 \alpha$ were detected in all ASST positive sera, which this finding strongly supported those reports. Interestingly, our study revealed that levels of anti-FceR $1 \alpha$ autoAbs were not statistically significant between ASST positive and ASST negative groups, as shown in Figures 1(b) and 2. Nevertheless, our finding was consistent with another report demonstrated that levels of autoAbs in CSU did not correlate with disease activity [29].

In conclusion, our ELISA detected significant levels of human IgG against FceR $1 \alpha$ in CSU sera, indicating that IgG anti-FceR $1 \alpha$ autoAbs are associated with CSU. Our finding suggested that IgG anti-FceR $1 \alpha$ could be a potential CSU biomarker and may predict outcomes of the new 
biologic treatment in the further study [34]. Importantly, our test might be an appropriate in vitro test, overcoming a limitation of ASST benefit for patients who cannot avoid antihistamine treatment.

\section{Data Availability}

The datasets generated and analyzed for the present study are available from the corresponding author on reasonable request.

\section{Disclosure}

The funding sponsors had no role in the design of the study, the collection, analysis and interpretation of the data, the writing of the manuscript, or the decision to publish the results.

\section{Conflicts of Interest}

The authors declare no conflict of interest.

\section{Authors' Contributions}

YS, KK, and DK conceived and designed the experiments. CS and TT performed the experiments. CS and TT prepared the data. KK and PT recruited patients. YS and CS analyzed the data and drafted the manuscript.

\section{Acknowledgments}

We would like to express appreciation to all volunteers, who kindly gave excellent cooperation. Special thanks are due to Ms. Sasipha Nuttawong, Ms. Nattacha Chanchaemsri, and Mr. Sumruay Pinkaew at Division of Allergy and Immunology, Department of Dermatology, Faculty of Medicine Siriraj Hospital, for their assistance in recruiting subjects and managing the database. This work is supported by the Faculty of Medicine Siriraj Hospital, Mahidol University, Bangkok, Thailand.

\section{Supplementary Materials}

Levels of hIgG anti-FceR $1 \alpha$ autoantibodies in sera from healthy, CSU, and autoimmune patients. Scatter plot represents levels of human IgG against anti-FceR $1 \alpha$ in healthy $(25 ; 400.2 \pm 38.90 \mathrm{ng} / \mathrm{ml})$, CSU patients $(116 ; 2,710 \pm 128.6$ $\mathrm{ng} / \mathrm{ml})$, and autoimmune patients (30;914.7 $\pm 127.3 \mathrm{ng} /$ $\mathrm{ml})(n$; mean $\pm \mathrm{SEM} \mathrm{ng} / \mathrm{ml})$. The cut-off line was set at $936.7 \mathrm{ng} / \mathrm{ml}$. Levels of hIgG anti-FceR $1 \alpha$ of most CSU were over cut-off point and significantly higher than that of autoimmune and healthy people. Levels of hIgG anti-FceR $1 \alpha$ of autoimmune patients were significantly higher than that of healthy people. (Supplementary Materials)

\section{References}

[1] S. S. Saini, M. Paterniti, K. Vasagar, S. P. Gibbons Jr., P. M. Sterba, and B. M. Vonakis, "Cultured peripheral blood mast cells from chronic idiopathic urticaria patients spontaneously degranulate upon IgE sensitization: relationship to expression of Syk and SHIP-2," Clinical Immunology, vol. 132, no. 3, pp. 342-348, 2009.

[2] B. M. Vonakis, S. Gibbons Jr., R. Sora, J. M. Langdon, and S. M. MacDonald, "Src homology 2 domain-containing inositol 5' phosphatase is negatively associated with histamine release to human recombinant histamine-releasing factor in human basophils," Journal of Allergy and Clinical Immunology, vol. 108, no. 5, pp. 822-831, 2001.

[3] P. Kolkhir, E. Borzova, C. Grattan, R. Asero, D. Pogorelov, and M. Maurer, "Autoimmune comorbidity in chronic spontaneous urticaria: a systematic review," Autoimmunity Reviews, vol. 16, no. 12, pp. 1196-1208, 2017.

[4] S. J. Bracken, S. Abraham, and A. S. MacLeod, "Autoimmune theories of chronic spontaneous urticaria," Frontiers in Immunology, vol. 10, p. 627, 2019.

[5] D. MacGlashan, "Autoantibodies to IgE and FcepsilonRI and the natural variability of spleen tyrosine kinase expression in basophils," Journal of Allergy and Clinical Immunology, vol. 143, no. 3, pp. 1100-1107, 2019.

[6] M. Hide, D. M. Francis, C. E. Grattan, J. Hakimi, J. P. Kochan, and M. W. Greaves, "Autoantibodies against the high-affinity IgE receptor as a cause of histamine release in chronic urticaria," New England Journal of Medicine, vol. 328, no. 22, pp. 1599-1604, 1993.

[7] N. Niimi, D. M. Francis, F. Kermani et al., "Dermal mast cell activation by autoantibodies against the high affinity $\operatorname{IgE}$ receptor in chronic urticaria," The Journal of Investigative Dermatology, vol. 106, no. 5, pp. 1001-1006, 1996.

[8] E. Fiebiger, D. Maurer, H. Holub et al., "Serum IgG autoantibodies directed against the alpha chain of Fc epsilon RI: a selective marker and pathogenetic factor for a distinct subset of chronic urticaria patients?," The Journal of Clinical Investigation, vol. 96, no. 6, pp. 2606-2612, 1995.

[9] L. J. Tong, G. Balakrishnan, J. P. Kochan, J. P. Kinét, and A. P. Kaplan, "Assessment of autoimmunity in patients with chronic urticaria," The Journal of Allergy and Clinical Immunology, vol. 99, no. 4, pp. 461-465, 1997.

[10] R. A. Sabroe, E. Fiebiger, D. M. Francis et al., "Classification of anti-FceRI and anti-IgE autoantibodies in chronic idiopathic urticaria and correlation with disease severity," The Journal of Allergy and Clinical Immunology, vol. 110, no. 3, pp. 492499, 2002.

[11] A. Puccetti, C. Bason, S. Simeoni et al., "In chronic idiopathic urticaria autoantibodies against FceRII/CD23 induce histamine release via eosinophil activation," Clinical and Experimental Allergy, vol. 35, no. 12, pp. 1599-1607, 2005.

[12] C. E. H. Grattan, T. B. Wallington, R. P. Warin, C. T. C. Kennedy, and J. W. Lbradfield, "A serological mediator in chronic idiopathic urticaria-a clinical, immunological and histological evaluation," The British Journal of Dermatology, vol. 114, no. 5, pp. 583-590, 1986.

[13] K. Kulthanan, S. Jiamton, T. Gorvanich, and S. Pinkaew, "Autologous serum skin test in chronic idiopathic urticaria: prevalence, correlation and clinical implications," Asian Pacific Journal of Allergy and Immunology, vol. 24, no. 4, pp. 201-206, 2006.

[14] O. Taskapan, A. Kutlu, and O. Karabudak, "Evaluation of autologous serum skin test results in patients with chronic idiopathic urticaria, allergic/non-allergic asthma or rhinitis and healthy people," Clinical and Experimental Dermatology, vol. 33, no. 6, pp. 754-758, 2008. 
[15] E. Fiebiger, F. Hammerschmid, G. Stingl, and D. Maurer, "Anti-FcepsilonRIalpha autoantibodies in autoimmunemediated disorders. Identification of a structure-function relationship," The Journal of Clinical Investigation, vol. 101, no. 1, pp. 243-251, 1998.

[16] S. Altrichter, V. Zampeli, A. Ellrich, K. Zhang, M. K. Church, and M. Maurer, "IgM and IgA in addition to IgG autoantibodies against $\mathrm{Fcc}_{\mathrm{C} I} \alpha$ are frequent and associated with disease markers of chronic spontaneous urticaria," Allergy, vol. 75, no. 12, pp. 3208-3215, 2020.

[17] S. Izaki, S. Toyoshima, T. Endo et al., "Differentiation between control subjects and patients with chronic spontaneous urticaria based on the ability of anti-IgE autoantibodies (AAbs) to induce FceRI crosslinking, as compared to anti-FceRI $\alpha$ AAbs," Allergology International, vol. 68, no. 3, pp. 342-351, 2019.

[18] M. Abd El-Azim and S. Abd El-Azim, "Chronic autoimmune urticaria: frequency and association with immunological markers," Journal of Investigational Allergology \& Clinical Immunology, vol. 21, no. 7, pp. 546-550, 2011.

[19] N. Bakos and M. Hillander, "Comparison of chronic autoimmune urticaria with chronic idiopathic urticaria," International Journal of Dermatology, vol. 42, no. 8, pp. 613-615, 2003.

[20] M. Caproni, W. Volpi, B. Giomi et al., "Chronic idiopathic and chronic autoimmune urticaria: clinical and immunopathological features of 68 subjects," Acta Dermato-Venereologica, vol. 84, no. 4, pp. 288-290, 2004.

[21] E. Nettis, P. Dambra, L. D'Oronzio et al., "Reactivity to autologous serum skintest and clinical features in chronic idiopathic urticaria," Clinical and Experimental Dermatology, vol. 27, no. 1, pp. 29-31, 2002.

[22] A. G. Vikramkumar, S. Kuruvila, and S. Ganguly, "Autologous serum skin test as an indicator of chronic autoimmune urticaria in a tertiary care hospital in South India," Indian Dermatology Online Journal, vol. 5, Suppl 2, pp. S87-S91, 2014.

[23] B. Ulambayar, Y. H. Chen, G. Y. Ban et al., "Detection of circulating IgG autoantibody to FcepsilonRIalpha in sera from chronic spontaneous urticaria patients," Journal of Microbiology, Immunology, and Infection, vol. 53, no. 1, pp. 141-147, 2020.

[24] A. Fusari, C. Colangelo, F. Bonifazi, and L. Antonicelli, "The autologous serum skin test in the follow-up of patients with chronic urticaria," Allergy, vol. 60, no. 2, pp. 256-258, 2005.

[25] R. Asero, M. Cugno, and A. Tedeschi, "Activation of blood coagulation in plasma from chronic urticaria patients with negative autologous plasma skin test," Journal of the European Academy of Dermatology and Venereology, vol. 25, no. 2, pp. 201-205, 2011.

[26] C. E. H. Grattan, D. M. Francis, M. Hide, and M. W. Greaves, "Detection of circulating histamine releasing autoantibodies with functional properties of anti-IgE in chronic urticaria," Clinical and Experimental Allergy, vol. 21, no. 6, pp. 695704, 1991.

[27] A. Kanani, S. D. Betschel, and R. Warrington, "Urticaria and angioedema," Allergy, Asthma and Clinical Immunology, vol. 14, Suppl 2, p. 59, 2018.

[28] A. P. Kaplan and K. Joseph, "Basophil secretion in chronic urticaria: autoantibody-dependent or not?," Journal of Allergy and Clinical Immunology, vol. 120, no. 3, pp. 729730, 2007.
[29] J. A. Eckman, R. G. Hamilton, L. M. Gober, P. M. Sterba, and S. S. Saini, "Basophil phenotypes in chronic idiopathic urticaria in relation to disease activity and autoantibodies," Journal of Investigative Dermatology, vol. 128, no. 8, pp. 19561963, 2008.

[30] S. Altrichter, H. J. Peter, D. Pisarevskaja, M. Metz, P. Martus, and M. Maurer, "IgE mediated autoallergy against thyroid peroxidase-a novel pathomechanism of chronic spontaneous urticaria?," PLoS One, vol. 6, no. 4, article e14794, 2011.

[31] M. F. Lee, T. M. Lin, S. W. Liu, and Y. H. Chen, "A rapid method of detecting autoantibody against FceRI $\alpha$ for chronic spontaneous urticaria," PLoS One, vol. 9, no. 10, article e109565, 2014

[32] P. Staubach, K. Onnen, A. Vonend et al., "Autologous whole blood injections to patients with chronic urticaria and a positive autologous serum skin test: a placebo-controlled trial," Dermatology, vol. 212, no. 2, pp. 150-159, 2006.

[33] K. Vasagar, B. M. Vonakis, L. M. Gober, A. Viksman, S. P. Gibbons, and S. S. Saini, "Evidence of in vivo basophil activation in chronic idiopathic urticaria," Clinical and Experimental Allergy, vol. 36, no. 6, pp. 770-776, 2006.

[34] S. A. Baioumy, M. M. Esawy, and M. A. Shabana, "Assessment of circulating FCeRIa in chronic spontaneous urticaria patients and its correlation with clinical and immunological variables," Immunobiology, vol. 223, no. 12, pp. 807-811, 2018. 